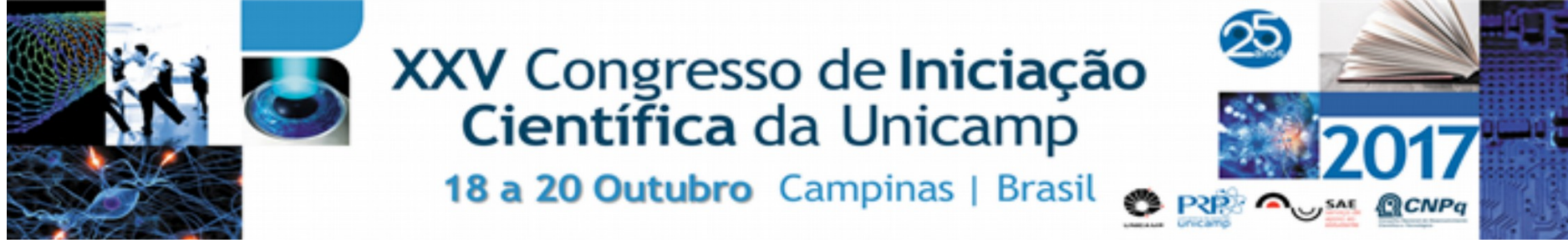

\title{
Detecção de Epilepsia por Aprendizagem de Máquina em Sinais de Eletroencefalograma
}

\section{Fabio T. K. Kubo*, Hélio Pedrini}

\section{Resumo}

Neste projeto, estudamos o uso da aprendizagem de máquina para o diagnóstico de epilepsia a partir de dados de eletroencefalograma (EEG) em três diferentes classificadores: máquinas de vetores de suporte (SVM), k-vizinhos mais próximos $(\mathrm{kNN})$ e random forests $(\mathrm{RF})$. O processo da aprendizagem incluiu um pré-processamento dos sinais utilizando a transformada discreta wavelet, a extração de um conjunto de onze características e, por fim, treinamento e teste de cada um dos classificadores. A classificação entre indivíduos epiléticos e saudáveis em um dos classificadores obteve uma acurácia de $98,0 \%$.

\section{Palavras-chave:}

Sinais EEG, aprendizado de máquina, epilepsia.

\section{Introdução}

A epilepsia é uma doença crônica caracterizada pela presença recorrente de crises epiléticas que são resultado de uma anormalidade na atividade elétrica cerebral.

A partir dos dados de EEG, podemos verificar a presença de epilepsia. Na forma tradicional, um especialista inspeciona, visualmente, os sinais de EEG e procura por padrões que indicam a presença de epilepsia, processo que possui alto custo, demanda tempo e está sujeito à falha humana. Outra forma é utilizar métodos automáticos, tais como aprendizado de máquina para analisar os sinais EEG, assunto que abordamos neste projeto.

O objetivo deste trabalho é estudar e comparar técnicas de aprendizado de máquina em sinais EEG.

\section{Resultados e Discussão}

Utilizamos a base de dados disponibilizada por Andrzejak et al. [1], que é composta por 500 amostras de EEG de 4096 medidas a frequência de 173,61 Hz.

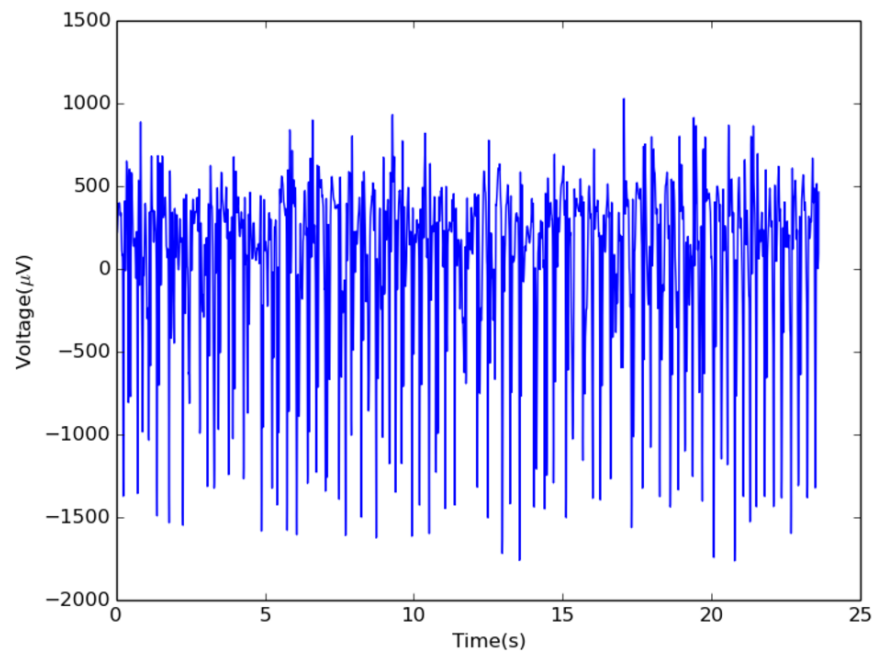

Figura 1. Visualização de sinal EEG de uma crise epilética.
Separamos as amostras de forma aleatória , tal que $70 \%$ são empregadas para treinamento e $30 \%$ para testes. Em cada uma das amostras, aplicamos a transformada wavelet db4 e extraímos um total de 11 características.

Em seguida, submetemos os dados a três diferentes classificadores: máquinas de vetores de suporte (SVM) com kernel polinomial, k-vizinhos mais próximos (kNN) e random forests (RF).

A partir da técnica descrita anteriormente, obtemos as taxas de acurácia mostradas na Tabela 1.

Tabela 1. Resultados dos classificadores.

\begin{tabular}{|c|c|}
\hline Classificador & Acurácia (\%) \\
\hline SVM & 98,0 \\
\hline kNN & 97,3 \\
\hline RF & 97,3 \\
\hline
\end{tabular}

\section{Conclusão}

Desenvolvemos um processo de aprendizagem supervisionada de máquina para auxiliar o diagnóstico de epilepsia, tornando a análise mais rápida e eficaz. As características extraídas dos dados se mostraram robustas para a classificação dos sinais EEG, atingindo uma taxa de 98,0\% de acurácia.

\footnotetext{
${ }^{1}$ Andrzejak, R.G.; Lehnertz, K.; Mormann, F.; Rieke, C.; David, P. e Elger, C. E. Indications of nonlinear deterministic and finite-dimensional structures in time series of brain electrical activity: Dependence on recording region and brain state. Physical Review E, 2001.

${ }^{2}$ Pereira, M. C. V. Avaliação de técnicas de pré-processamento de sinais do EEG para detecção de eventos epileptogênicos utilizando redes neurais artificiais. Em Universidade Federal de Santa Catarina, 2003.

${ }^{3}$ Wulsin, D. F.; Gupta, J. R; Mani, R.; Blanco, J. A. e Litt, B. Modeling electroencephalography waveforms with semi-supervised deep belief nets: fast classification and anomaly measurement. IOPscience, 2011.

${ }^{4}$ Adeli, H.; Zhou Z. e Dadmehr, N. Analysis of EEG records in an epileptic patient using wavelet transform. Elsevier, 2002.
} 